\title{
On the Topology of the Space of Contact Structures on Torus Bundles
}

\author{
Hansjörg Geiges and Jesús Gonzalo
}

\begin{abstract}
We prove the existence of essential loops in the space of contact structures on torus bundles over the circle.
\end{abstract}

\section{Introduction}

Very little seems to be known about the topology of the space $\Xi(M)$ of contact structures on a given manifold $M$. (There is, as yet, no standard notation for that space. Since $\xi$ is a customary notation for contact structures, the letter $\Xi$ seems an apt choice.) Some general results about the structure of $\Xi$ can be found in [10, Section 2.4] and [13] (the latter has to be read with a certain amount of caution; see Mathematical Reviews 83k:58018). For our purposes we only need to observe that $\Xi(M)$ is an open subset (in the $C^{k}$-topology for $k \geq 1$ ) of the Grassmannian manifold of all codimension one subbundles of the tangent bundle $T M$.

There also appear to be few results concerning $\Xi(M)$ for specific $M$. One such result is due to Eliashberg. Let $\xi_{0}=\operatorname{ker}(x d y-y d x+z d t-t d z)$ be the standard contact structure on $S^{3} \subset \mathbb{R}^{4}$ and denote by $\Xi_{0}\left(S^{3}, p_{0}\right)$ the subspace of $\Xi\left(S^{3}\right)$ which consists of contact structures isotopic to $\xi_{0}$ and with $\xi\left(p_{0}\right)=$ $\xi_{0}\left(p_{0}\right)$ for some fixed $p_{0} \in S^{3}$. In [2] Eliashberg has shown that $\Xi_{0}\left(S^{3}, p_{0}\right)$ is contractible.

This result can be rephrased as follows. Write Diff $p_{0}^{+}\left(S^{3}\right)$ for the group of orientation preserving diffeomorphisms of $S^{3}$ that fix the contact plane $\xi_{0}\left(p_{0}\right)$, and denote by $\operatorname{Cont}\left(S^{3}, \xi_{0}\right)$ the subgroup of $\operatorname{Diff}_{p_{0}}^{+}\left(S^{3}\right)$ consisting of diffeomorphisms which preserve $\xi_{0}$. Then Eliashberg's theorem says that the inclusion of $\operatorname{Cont}\left(S^{3}, \xi_{0}\right)$ in $\operatorname{Diff}_{p_{0}}^{+}\left(S^{3}\right)$ is a homotopy equivalence, cf. Section 3 Giroux [9] gives results about the connected components of other such contactomorphism groups.

The present paper is concerned with the topology of the space of contact structures on $T^{2}$-bundles over $S^{1}$. Specifically, we provide an essentially elementary argument showing the fundamental group of these spaces (with base point specified below) to contain an infinite cyclic subgroup. The basic techniques of the proof are classical (Gray stability and contact Hamiltonians), but a key ingredient is the recent work of Giroux [8] and Kanda [11] on the classification 
of contact structures on the 3-torus. Throughout we assume contact structures to be coorientable, so they can be defined by global contact forms.

For results about related homotopical questions in symplectic topology see for instance [1] and 14.

\section{Contact geometry of the 3-torus}

Let $(x, y, \theta)$ be standard angular coordinates on $T^{3}=(\mathbb{R} / 2 \pi \mathbb{Z})^{3}$. Define, for $n \in \mathbb{N}$,

$$
\alpha_{s}^{n}=\cos (n \theta-2 \pi s) d x-\sin (n \theta-2 \pi s) d y, \quad s \in[0,1],
$$

and $\zeta_{s}^{n}=\operatorname{ker} \alpha_{s}^{n}$. We shall abbreviate $\zeta_{s}^{1}$ to $\zeta_{s}$.

Giroux and Kanda have shown that the $\zeta_{0}^{n}$ provide a complete list, up to diffeomorphism, of so-called tight contact structures on the 3-torus (see 2] for the definition of 'tight'). Notice, however, that the isotopy classification is more subtle. According to Eliashberg and Polterovich [4, for $\phi \in \operatorname{SL}(3, \mathbb{Z}) \subset$ $\operatorname{Diff}^{+}\left(T^{3}\right)$ the contact structures $\phi_{*} \zeta_{0}$ and $\zeta_{0}$ are isotopic if and only if $\phi$ fixes the subspace $\mathbb{Z}^{2} \oplus 0$ of the first homology group. Taken together, these results yield complete information about the set of connected components of $\Xi\left(T^{3}\right)$, it being known that $\pi_{0}\left(\operatorname{Diff}^{+}\left(T^{3}\right)\right)=\mathrm{SL}(3, \mathbb{Z})$.

The following is our main result about the topology of $\Xi\left(T^{3}\right)$. Below we shall formulate corresponding statements for all other torus bundles over $S^{1}$.

Proposition 1. For each $n \in \mathbb{N}$ the fundamental group $\pi_{1}\left(\Xi\left(T^{3}\right), \zeta_{0}^{n}\right)$ based at $\zeta_{0}^{n}$ contains an infinite cyclic subgroup, generated by the loop $\left\{\zeta_{s}^{n}: 0 \leq s \leq 1\right\}$.

Together with the long homotopy exact sequence of the next section and the known results about the homotopy type of $\operatorname{Diff}\left(T^{3}\right)$ this can be related to the topology of contactomorphism groups.

Notice that the loop $\left\{\xi_{s}\right\}$ is defined by a linear circle of contact forms,

$$
\xi_{s}=\operatorname{ker}\left(\cos (2 \pi s) \alpha_{0}+\sin (2 \pi s) \alpha_{1 / 4}\right) .
$$

A pair of contact forms $\left(\alpha_{0}, \alpha_{1 / 4}\right)$ with this property that any non-trivial linear combination of these forms is again a contact form was called a contact circle in [6, 7, and it is natural to ask what can be said about such contact circles as elements of $\pi_{1}(\Xi(M))$. In $[7$ it is shown that contact circles exist on all closed, orientable 3-manifolds.

Our proof of Proposition 1 makes essential use of the diffeomorphism classification of contact structures on $T^{3}$. It is worth emphasising that the analogue of Proposition 1 for more general torus bundles over the circle, formulated in Section [5 below, rests equally on the classification of contact structures on $T^{3}$, and not on the classification of contact structures on these spaces themselves. Again the essential loop is a contact circle.

Clearly, if a contact circle $\left(\beta_{1}, \beta_{2}\right)$ extends to a contact sphere $\left(\beta_{1}, \beta_{2}, \beta_{3}\right)$, i.e. a triple of contact forms such that any non-trivial linear combination is a contact form, then $\left(\beta_{1}, \beta_{2}\right)$ defines the trivial element in $\pi_{1}(\Xi(M))$. 
On the other hand, evaluation at a point $p \in M$ and identification of cooriented radial 2-planes in $\mathbb{R}^{3}$ with $S^{2}$ defines a diffeomorphism

$$
\begin{aligned}
& \mathbb{R}^{3} \supset S^{2} \longrightarrow S^{2} \\
&\left(\lambda_{1}, \lambda_{2}, \lambda_{3}\right) \longmapsto \\
& \operatorname{ker}\left(\lambda_{1} \alpha_{1}+\lambda_{2} \alpha_{2}+\lambda_{3} \alpha_{3}\right)(p),
\end{aligned}
$$

so a contact sphere always maps to the generator of $\pi_{2}\left(S^{2}\right)$ under this evaluation map and hence defines an element of infinite order in $\pi_{2}(\Xi(M))$. Contact spheres exist, for instance, on the connected sum of left-quotients of $\mathrm{SU}(2)$ and copies of $S^{1} \times S^{2}$, see [7] Prop. 5.7]. This does not contradict Eliashberg's result about the contractibility of $\Xi_{0}\left(S^{3}, p_{0}\right)$, of course, since in $\Xi\left(S^{3}\right)$ we do not fix the contact plane at a point.

\section{A homotopy exact sequence}

The following considerations rest on the well-known concepts of Gray stability and contact Hamiltonians, cf. [12, [5]. We only recall briefly the facts that we shall use:

Gray stability: Given a smooth family of contact structures $\xi_{t}, t \in[0,1]$, on a closed manifold $M$, there is a canonically defined time-dependent vector field $X_{t}$ tangent to $\xi_{t}$ whose flow $\psi_{t}$ satisfies $\psi_{t *} \xi_{0}=\xi_{t}$ (that is, the differential $T \psi_{t}$ maps $\xi_{0}$ to $\xi_{t}$ ). Given a family of contact forms $\alpha_{t}$ defining $\xi_{t}$, with Reeb vector field $R_{t}$, this vector field is determined by the equations $\alpha_{t}\left(X_{t}\right)=0$ and $i_{X_{t}} d \alpha_{t}=\mu_{t} \alpha_{t}-\dot{\alpha}_{t}$, with the function $\mu_{t}$ determined by $\mu_{t}=\dot{\alpha}_{t}\left(R_{t}\right)$, and with $\dot{\alpha}_{t}$ denoting the time derivative of $\alpha_{t}$. This $X_{t}$ is independent of the choice of $\alpha_{t}$, even though $\mu_{t}$ does depend on that choice.

Contact Hamiltonians: Given a smooth family of functions $H_{t}$ on a contact manifold $(M, \xi=\operatorname{ker} \alpha)$, a time-dependent vector field $X_{t}$ whose flow preserves $\xi$ is defined by

$$
X_{t}=H_{t} R+Y_{t}
$$

where $R$ is the Reeb vector field of $\alpha$ and $Y_{t}$ is determined by

$$
\alpha\left(Y_{t}\right)=0
$$

and

$$
i_{Y_{t}} d \alpha=d H_{t}(R) \alpha-d H_{t} .
$$

Indeed, the infinitesimal condition $L_{X_{t}} \alpha=\rho_{t} \alpha$ is equivalent to the equations above if we require $H_{t}=\alpha\left(X_{t}\right)$. Put differently, a time-dependent vector field $X_{t}$ whose flow preserves $\xi=\operatorname{ker} \alpha$ is completely determined by $H_{t}=\alpha\left(X_{t}\right)$, the Hamiltonian function of $X_{t}$.

Let $\left(M, \xi_{0}\right)$ be a closed contact manifold. Write $\operatorname{Diff}_{0}(M)$ for the identity component of the diffeomorphism group of $M$ and $\operatorname{Cont}_{0}\left(M, \xi_{0}\right)$ for its subgroup of contactomorphisms, i.e.

$$
\operatorname{Cont}_{0}\left(M, \xi_{0}\right)=\left\{\phi \in \operatorname{Diff}_{0}(M): \phi_{*} \xi_{0}=\xi_{0}\right\} .
$$


We have an obvious inclusion $i: \operatorname{Cont}_{0}\left(M, \xi_{0}\right) \rightarrow \operatorname{Diff}_{0}(M)$ and, by Gray stability, a surjection

$$
\begin{aligned}
\sigma: \operatorname{Diff}_{0}(M) & \longrightarrow \Xi_{0}(M) \\
\phi & \longmapsto \phi_{*} \xi_{0},
\end{aligned}
$$

where $\Xi_{0}(M)$ denotes the component of $\Xi(M)$ containing $\xi_{0}$.

The following is a well-known (folklore) result:

Proposition 2. The map $\sigma$ has the homotopy lifting property with respect to cubes $I^{n}=[0,1]^{n}$ and smooth homotopies. Hence there is an exact sequence of homotopy groups

$$
\ldots \stackrel{\Delta}{\longrightarrow} \pi_{k}\left(\text { Cont }_{0}\right) \stackrel{i_{\#}}{\longrightarrow} \pi_{k}\left(\text { Diff }_{0}\right) \stackrel{\sigma_{\#}}{\longrightarrow} \pi_{k}\left(\Xi_{0}\right) \stackrel{\Delta}{\longrightarrow} \pi_{k-1}\left(\text { Cont }_{0}\right) \stackrel{i_{\#}}{\longrightarrow} \ldots
$$

Proof. We only need to prove the first statement. The usual proof that a Serre fibration yields an exact sequence of homotopy groups then still applies to give the desired conclusion.

This means we are given a commutative diagram

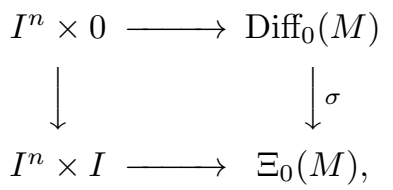

with the bottom map smooth in the $I$-factor; more precisely, a family $\xi_{s, t}$ of contact structures on $M$, continuous in $s \in I^{n}$, smooth in $t \in I$, and with all $t$-derivatives continuous in $s$. We also have a family $\phi_{s, 0} \in \operatorname{Diff}_{0}(M)$ with $\left(\phi_{s, 0}\right)_{*} \xi_{0}=\xi_{s, 0}$, and the aim is to find a lifting $I^{n} \times I \rightarrow \operatorname{Diff}_{0}(M)$.

By Gray stability (with an added parameter $s$ ) one finds a family of vector fields $X_{s, t}$, continuous in $s$ and smooth in $t$, such that the flow of the $t$-dependent vector field $X_{s, t}$ defines a family of diffeotopies $\psi_{s, t}$ which is continuous in $s$ and satisfies $\left(\psi_{s, t}\right)_{*} \xi_{s, 0}=\xi_{s, t}$. Set $\phi_{s, t}=\psi_{s, t} \circ \phi_{s, 0}$. Then $\left(\phi_{s, t}\right)_{*} \xi_{0}=\xi_{s, t}$, so $\phi_{s, t}$ is the desired lift of $\xi_{s, t}$.

\section{Proof of Proposition 1}

We only show that $\left\{\zeta_{s}=\zeta_{s}^{1}\right\}$ is non-zero in $\pi_{1}$; a completely analogous argument applies to any multiple of this loop and to general $n$.

Our proof is by contradiction. Assume $\left\{\zeta_{s}\right\}$ defines the trivial element in $\pi_{1}\left(\Xi\left(T^{3}\right), \zeta_{0}\right)$. Write $\pi$ for the canonical submersion

$$
T^{2} \times \mathbb{R} \longrightarrow T^{2} \times \mathbb{R} / 2 \pi \mathbb{Z} \equiv T^{3}
$$

and set $\widetilde{\zeta}_{s}=\pi^{*} \zeta_{s}$ for each $s \in[0,1]$. Then $\left\{\widetilde{\zeta}_{s}\right\}$ would define the trivial element in $\pi_{1}\left(\Xi_{0}^{\mathbb{Z}}\left(T^{2} \times \mathbb{R}\right)\right)$, where $\Xi_{0}^{\mathbb{Z}}\left(T^{2} \times \mathbb{R}\right)$ denotes the connected component containing $\widetilde{\zeta}_{0}$ of the space of contact structures on $T^{2} \times \mathbb{R}$ which are invariant under shifts by $2 \pi$ in $\mathbb{R}$-direction. 
Proposition $\left[2\right.$ applies to $T^{2} \times \mathbb{R}$ if we replace $\Xi_{0}$ by $\Xi_{0}^{\mathbb{Z}}\left(T^{2} \times \mathbb{R}\right)$ and $\operatorname{Diff}_{0}$ by $\operatorname{Diff}_{0}^{\mathbb{Z}}\left(T^{2} \times \mathbb{R}\right)$, the identity component of the $\mathbb{Z}$-equivariant diffeomorphisms of $T^{2} \times \mathbb{R}$, i.e.

$$
\operatorname{Diff}_{0}^{\mathbb{Z}}\left(T^{2} \times \mathbb{R}\right)=\left\{\phi \in \operatorname{Diff}\left(T^{2} \times \mathbb{R}\right): \phi(x, y, \theta+2 \pi)=\phi(x, y, \theta)+(0,0,2 \pi)\right\}_{0},
$$

for the $\mathbb{Z}$-invariance resp. -equivariance guarantees that the vector field $X_{s, t}$ used in the proof of Proposition 2 still integrates to a global flow.

We would then have a homotopy $\left\{\widetilde{\zeta}_{s, t}\right\}$ rel $\{0,1\}$ of $\left\{\widetilde{\zeta}_{s}\right\}$ to the constant loop $\left\{\widetilde{\zeta}_{0}\right\}$. We may assume that $\widetilde{\zeta}_{s, t}$ is smooth in $t$ (by smoothing a homotopy which is constant near $t=0,1)$.

Define a diffeomorphism

$$
\begin{aligned}
\widetilde{\phi}_{s}: T^{2} \times \mathbb{R} & \longrightarrow T^{2} \times \mathbb{R} \\
(x, y, \theta) & \longmapsto(x, y, \theta+2 \pi s),
\end{aligned}
$$

so that $\widetilde{\phi}_{s *} \widetilde{\zeta}_{0}=\widetilde{\zeta}_{s, 0}=\widetilde{\zeta}_{0}$. By Proposition 2 we find a path $\psi(s)$ in $\operatorname{Cont}_{0}\left(T^{2} \times\right.$ $\left.\mathbb{R}, \widetilde{\zeta}_{0}\right)$ that joins $\widetilde{\phi}_{0}$ with $\widetilde{\phi}_{1}$.

Let $X_{s}$ be the time-dependent vector field that integrates to $\psi(s)$ at time $s$. Choose a contact form $\widetilde{\alpha}_{0}$ representing $\widetilde{\zeta}_{0}$ and set $H_{s}=\widetilde{\alpha}_{0}\left(X_{s}\right)$. Since the flow of $X_{s}$ preserves $\widetilde{\zeta}_{0}$, the Hamiltonian vector field of $H_{s}$ coincides with $X_{s}$. The time-one-map of this Hamiltonian flow is $\widetilde{\phi}_{1}$. During the diffeotopy, the image of $T^{2} \times[0,2 \pi]$ under $\psi(s)$ stays above $T^{2} \times\{-2 \pi(k-1)\}$ for some $k \in \mathbb{N}$ sufficiently large.

Now let $\chi(\theta)$ be a smooth function with $\chi(\theta) \equiv 0$ for $\theta \leq-2 \pi k$ and $\chi(\theta) \equiv 1$ for $\theta \geq-2 \pi(k-1)$, and set $\bar{H}_{s}(x, y, \theta)=H_{s}(x, y, \theta) \chi(\theta)$. The Hamiltonian vector field $\bar{X}_{s}$ of $\bar{H}_{s}$ still integrates to a global flow, and the time-one-map of this flow defines a $\widetilde{\zeta}_{0}$-preserving diffeomorphism

$$
T^{2} \times[-2 \pi k, 0] \longrightarrow T^{2} \times[-2 \pi k, 2 \pi]
$$

But this would imply that the contact structures $\zeta^{k}$ and $\zeta^{k+1}$ on $T^{3}$ are diffeomorphic, contradicting the work of Giroux and Kanda. This contradiction proves the proposition.

Here is an alternative proof of Proposition 1 based on the work of EliashbergHofer-Salamon [3. Again arguing by contradiction, we assume that $\left\{\zeta_{s}\right\}$ is trivial in $\pi_{1}\left(\Xi\left(T^{3}\right), \zeta_{0}\right)$. Consider the double cover

$$
T_{4 \pi}^{3} \equiv T^{2} \times \mathbb{R} / 4 \pi \mathbb{Z} \longrightarrow T^{2} \times \mathbb{R} / 2 \pi \mathbb{Z} \equiv T^{3},
$$

and write $\zeta_{s}^{\prime}$ for the lift of $\zeta_{s}$. Then $\left\{\zeta_{s}^{\prime}\right\}$ is also a contractible loop in the corresponding space of contact structures.

The same argument as before yields a contact isotopy of $\left(T_{4 \pi}^{3}, \zeta_{0}^{\prime}\right)$, beginning at the identity and ending with a shift by $2 \pi$ in $\theta$-direction. In particular, this contact isotopy would move the pre-Lagrangian torus (see 3]) $T^{2} \times\{0\}$ to $T^{2} \times\{2 \pi\}$ and therefore separate it completely from itself. This would contradict 
3. Thm. 3.8.3], which uses a Floer homology argument to provide a lower bound for the number of intersection points between a pre-Lagrangian submanifold $\Lambda_{0}$ and the image $\Lambda_{1}^{\prime}$ of a Legendrian submanifold $\Lambda_{1} \subset \Lambda_{0}$ under a contact isotopy (The conditions $O_{1}$ and $O_{2}$ of that theorem are satisfied in the present context).

\section{Contact circles on torus bundles}

We now extend Proposition 1 to all torus bundles over the circle. For $A \in \mathrm{SL}_{2} \mathbb{Z}$, write $M_{A}$ for the quotient of $T^{2} \times \mathbb{R}$ under the transformation

$$
\widetilde{A}:\left(\left(\begin{array}{l}
x \\
y
\end{array}\right), \theta\right) \longmapsto\left(A\left(\begin{array}{l}
x \\
y
\end{array}\right), \theta+2 \pi\right) .
$$

On each $M_{A}$ there is a family of contact structures $\zeta^{n}, n \in \mathbb{N}$, characterised up to fibre preserving isotopy by the property that it descends from a contact structure on $\mathbb{R}^{2} \times \mathbb{R}$ of the form

$$
\cos f(\theta) d x-\sin f(\theta) d y=0,
$$

invariant under the transformation $\widetilde{A}$, and with

$$
2(n-1) \pi<\sup _{\theta \in \mathbb{R}}(f(\theta+1)-f(\theta)) \leq 2 n \pi,
$$

see 8 .

Proposition 3. For each $A \in \mathrm{SL}_{2} \mathbb{Z}$ and $n \in \mathbb{N}$, the fundamental group of $\Xi\left(M_{A}\right)$ based at $\zeta^{n}$ contains an element of infinite order.

To prove this proposition it is convenient to use the Thurston geometries adapted to the different choices of $A$, as done previously in [7. Rather than giving a case by case proof, we illustrate the method by a particular example which contains all the ideas required for the general argument.

Assume that trace $A \geq 3$. Then $A$ is conjugate in $\mathrm{GL}_{2} \mathbb{R}$ to a matrix of the form

$$
A^{\prime}=\left(\begin{array}{cc}
e^{\gamma} & 0 \\
0 & e^{-\gamma}
\end{array}\right)
$$

and $M_{A}$ is modelled on the solvable Lie group $\mathrm{Sol}^{3}$ (the inhomogeneous Lorentz group), cf. [7. This means that we can write $M_{A}$ as a quotient of $\mathbb{R}^{3}$ under a group of transformations $\Gamma$ generated by

$$
\begin{aligned}
& (x, y, \theta) \longmapsto\left(x+a_{1}, y+b_{1}, \theta\right) \\
& (x, y, \theta) \longmapsto\left(x+a_{2}, y+b_{2}, \theta\right) \\
& (x, y, \theta) \longmapsto\left(e^{\gamma} x, e^{-\gamma} y, \theta+\gamma\right),
\end{aligned}
$$

where the vectors

$$
\left(\begin{array}{c}
a_{1} \\
b_{1}
\end{array}\right),\left(\begin{array}{c}
a_{2} \\
b_{2}
\end{array}\right)
$$


generate a lattice $\Lambda$ in $\mathbb{R}^{2}$ invariant under $A^{\prime}$.

Now define, for $n \in \mathbb{N}$,

$$
\alpha_{s}^{n}=\cos \left(\frac{2 \pi n \theta}{\gamma}-2 \pi s\right) e^{-\theta} d x-\sin \left(\frac{2 \pi n \theta}{\gamma}-2 \pi s\right) e^{\theta} d y, \quad s \in[0,1],
$$

and set $\zeta_{s}^{n}=\operatorname{ker} \alpha_{s}^{n}$. This defines a contact structure on $\mathbb{R}^{3}$ that descends to $M_{A}$. Up to fibre preserving isotopy, $\zeta_{0}^{n}$ is equal to the $\zeta^{n}$ studied by Giroux. Write $\widetilde{\zeta}_{0}$ for the lift of $\zeta_{0}=\zeta_{0}^{1}$ to $T^{2} \times \mathbb{R}$.

Denote by $\Xi_{0}^{A^{\prime}}\left(T^{2} \times \mathbb{R}\right)$ the connected component of $\widetilde{\zeta}_{0}$ in the space of contact structures on $T^{2} \times \mathbb{R}$ invariant under the transformation

$$
\widetilde{A}^{\prime}:(x, y, \theta) \longmapsto\left(e^{\gamma} x, e^{-\gamma} y, \theta+\gamma\right),
$$

where we think of $T^{2}$ as the quotient of $\mathbb{R}^{2}$ under the lattice $\Lambda$. Write Diff $A_{0}^{A^{\prime}}\left(T^{2} \times\right.$ $\mathbb{R})$ for the identity component of the diffeomorphisms of $T^{2} \times \mathbb{R}$ that commute with $\widetilde{A}^{\prime}$.

Then the argument in the proof of Proposition 1 goes through as before, with $\Xi_{0}^{\mathbb{Z}}\left(T^{2} \times \mathbb{R}\right)$ replaced by $\Xi_{0}^{A^{\prime}}\left(T^{2} \times \mathbb{R}\right)$, and $\operatorname{Diff}_{0}^{\mathbb{Z}}\left(T^{2} \times \mathbb{R}\right)$ by $\operatorname{Diff}_{0}^{A^{\prime}}\left(T^{2} \times \mathbb{R}\right)$. Assuming that $\left\{\zeta_{s}\right\}$ was trivial in $\pi_{1}\left(\Xi\left(M_{A}\right), \zeta_{0}\right)$, we obtain a $\widetilde{\zeta}_{0}$-preserving diffeomorphism

$$
T^{2} \times[-k \gamma, 0] \longrightarrow T^{2} \times[-k \gamma, \gamma] .
$$

On $T^{2} \times\{l \gamma\}, l \in \mathbb{Z}$, the contact form $\alpha_{0}^{1}$ restricts to $e^{-l \gamma} d x$, so the characteristic foliation on these tori is always given by $d x=0$. Since the characteristic foliation on a surface determines the germ of the contact structure along that surface, we can use the identity map on $T^{2}$ to glue the ends of $T^{2} \times[-k \gamma, \varepsilon \gamma]$, with $\varepsilon \in\{0,1\}$, and obtain a contact structure on $T^{3}$, diffeomorphic to the standard structure $\zeta^{k}$ of $T^{3}$ for $\varepsilon=0$, and $\zeta^{k+1}$ for $\varepsilon=1$. The diffeomorphism above would induce a diffeomorphism between $\zeta^{k}$ and $\zeta^{k+1}$ on $T^{3}$, contradicting once again the work of Giroux and Kanda.

\section{References}

[1] M. Abreu and D. McDuff, Topology of symplectomorphism groups of rational ruled surfaces, J. Amer. Math. Soc. 13 (2000), 971-1009.

[2] Y. Eliashberg, Contact 3-manifolds twenty years since J. Martinet's work, Ann. Inst. Fourier (Grenoble) 42 (1992), 165-192.

[3] Y. Eliashberg, H. Hofer, and D. Salamon, Lagrangian intersections in contact geometry, Geom. Funct. Anal. 5 (1995), 244-269.

[4] Y. Eliashberg and L. Polterovich, New applications of Luttinger's surgery, Comment. Math. Helv. 69 (1994), 512-522.

[5] H. Geiges, Contact geometry, in: Handbook of Differential Geometry vol. 2 (F.J.E. Dillen and L.C.A. Verstraelen, eds.), to appear. 
[6] H. Geiges and J. Gonzalo, Contact geometry and complex surfaces, Invent. Math. 121 (1995), 147-209.

[7] H. Geiges and J. Gonzalo, Contact circles on 3-manifolds, J. Differential Geom. 46 (1997), 236-286.

[8] E. Giroux, Une infinité de structures de contact tendues sur une infinité de variétés, Invent. Math. 135 (1999), 789-802.

[9] E. Giroux, Sur les transformations de contact au-dessus des surfaces, in: Essays on Geometry and Related Topics, Mémoires dédiés à André Haefliger (E. Ghys et al., eds.), Monogr. Enseign. Math. 38, vol. 2, Genève (2001), 329-350.

[10] R.S. Hamilton, The inverse function theorem of Nash and Moser, Bull. Amer. Math. Soc. (N.S.) 7 (1982), 65-222.

[11] Y. Kanda, The classification of tight contact structures on the 3-torus, Comm. Anal. Geom. 5 (1997), 413-438.

[12] P. Libermann and C.-M. Marle, Symplectic Geometry and Analytical Mechanics, Reidel Publishing Company, Dordrecht, 1987.

[13] L. Maxim Răileanu, The manifold of contact structures on a compact manifold, Rend. Mat. (7) 2 (1982), 117-122.

[14] P. Seidel, $\pi_{1}$ of symplectic automorphism groups and invertibles in quantum homology rings, Geom. Funct. Anal. 7 (1997), 1046-1095.

Hansjörg Geiges

Mathematisches Institut

Universität zu Köln

Weyertal 86-90

50931 Köln

Germany

e-mail: geiges@math.uni-koeln.de
Jesús Gonzalo

Departamento de Matemáticas

Universidad Autónoma de Madrid

28049 Madrid

Spain

e-mail: jesus.gonzalo@uam.es 